\title{
Impedance spectroscopy of organic hetero-layer OLEDs as a probe for charge carrier injection and device degradation
}

\author{
Stefan Nowy, Wei Ren, Julia Wagner, Josef A. Weber, and Wolfgang Brütting \\ Institute of Physics, University of Augsburg, Germany
}

\begin{abstract}
Impedance spectroscopy (IS) is a powerful method for characterizing the electrical properties of materials and their interfaces. In this study we use IS to investigate the charge carrier injection properties of different anodes and anode treatments in bottom-emitting organic light-emitting diodes (OLEDs). These are ITO-based (indium tin oxide) hetero-layer devices with TPD (N,N'-diphenyl-N,N'-bis(3-methylphenyl)-1,1'-biphenyl-4,4-diamine) as hole transporting layer (HTL) and $\mathrm{Alq}_{3}$ (tris-(8-hydroxyquinoline) aluminum) as emission and electron transporting layer (EML and ETL, respectively). A detailed analysis of the capacitance as function of frequency and DC bias yields information about trapped and interfacial charges as well as the dynamics of injected charges. Furthermore, we use IS to study degradation processes in OLEDs.
\end{abstract}

Keywords: organic light-emitting diodes, impedance spectroscopy, charge carrier injection, device degradation

\section{INTRODUCTION}

Impedance spectroscopy is a widely used tool for inorganic semiconductors and also for studies of solid-liquid interfaces. ${ }^{1,2}$ It is a powerful method of characterizing many of the electrical properties of materials and their interfaces. It is employed to investigate the dynamics of bound or mobile charge in the bulk or interfacial regions, with the great advantage of being a non-destructive analysis. In literature, IS has been successfully applied to a variety of issues for organic electronics. Capacitance-voltage $(C-V)$ measurements were used to extract the acceptor dopant concentration in poly-(p-phenylene-vinylene) (PPV) Schottky diodes. ${ }^{3}$ Additionally the energetic depth and the density of states created by the doping of PPV were extracted from temperature dependent investigations. ${ }^{4}$ The trap and transport states distributions for hole transport in PPV were determined by transient capacitance measurements. ${ }^{5}$ Furthermore, the dynamics of injected charge carriers was investigated under space charge limited current (SCLC) conditions, i.e. under forward bias, for PPV derivatives ${ }^{6}$ and $\mathrm{Alq}_{3}{ }^{7}$ More recently, it was shown that $C-V$ analysis in single carrier devices can also yield information about injection barriers and the built-in voltage resulting from different metal contacts. ${ }^{8}$ Going to double carrier devices, such as organic light-emitting diodes (OLEDs) or solar cells, additional features related to the dynamics of electron-hole pairs can be monitored using IS. It was shown, e.g., that charge carrier recombination leads to the appearance of negative capacitance. ${ }^{9}$

In hetero-layer devices, as discussed in this study, qualitatively new features can arise from the presence of an internal organic-organic interface. It was shown earlier by our group that in OLEDs comprising a hetero-interface between a hole transporting and an electron transporting material a fixed interfacial charge can be detected by applying capacitance-voltage measurements. ${ }^{10}$ In this paper, we will demonstrate how IS of this phenomenon can be extended to obtain information about the dynamics of injected charges and about changes at interfaces caused by device degradation under electrical operation.

Further author information: S.N.: E-mail: stefan.nowy@physik.uni-augsburg.de W.B.: E-mail: wolfgang.bruetting@physik.uni-augsburg.de 
Table 1: Four anode modifications of the OLED in fig. 1 used in this paper.

\begin{tabular}{|c||c|c|c|c|}
\hline type & $(1)$ & $(2)$ & $(3)$ & $(4)$ \\
\hline ITO plasma treatment & $\mathrm{O}_{2}$ & $\mathrm{O}_{2}$ & $\mathrm{O}_{2}$ & $\mathrm{Ar}$ \\
\hline interlayer & - & thin $\mathrm{F}_{4}$-TCNQ & $20 \mathrm{~nm}$ PEDOT:PSS & $10 \mathrm{~nm} \mathrm{WO}_{3}$ \\
\hline
\end{tabular}

\section{EXPERIMENTAL}

In this paper we use a well-known OLED stack comprising a hole and an electron transporting compartment, respectively (fig. 1). The substrates for the bottom-emitting OLEDs consist of glass with a patterned, $140 \mathrm{~nm}$ thick indium tin oxide (ITO) layer, which are cleaned with standard procedures. The ITO layer can optionally be $\mathrm{O}_{2}$ or Ar plasma treated to further clean the substrates and modify its workfunction. Together with an optional interlayer the ITO forms the anode, which injects holes into the OLED. Four different types of anodes are compared here: (1) $\mathrm{O}_{2}$ plasma treated ITO without interlayer, (2) with a thin $\mathrm{F}_{4}$-TCNQ interlayer, (3) with a $20 \mathrm{~nm}$ interlayer of poly(3,4)-ethylendioxythiophene doped with poly(styrene sulfonate) (PEDOT:PSS), and (4) Ar plasma treated ITO with a $10 \mathrm{~nm} \mathrm{WO}_{3}$ interlayer (table 1). As hole transport layer (HTL) $80 \mathrm{~nm}$ of N,N'-diphenyl-N,N'-bis(3-methylphenyl)-1,1'-biphenyl-4,4-diamine (TPD) is used. The electron conducting compartment consists of $80 \mathrm{~nm}$ electron transport layer (ETL) tris-(8-hydroxyquinoline) aluminum $\left(\mathrm{Alq}_{3}\right), 15 \mathrm{~nm}$ $\mathrm{Ca}$, and $100 \mathrm{~nm} \mathrm{Al}$. The emission zone is located at the $\mathrm{TPD} / \mathrm{Alq}_{3}$-interface (approximately $10 \mathrm{~nm}$ wide).

For type (3) devices the polymer PEDOT:PSS (Baytron P from H. C. Starck) is spincast onto the cleaned substrate and dried on a hot plate in a cleanroom. All other organic and metal(-oxide) layers are deposited through shadow masks in a high vacuum chamber (base pressure $<3 \times 10^{-7} \mathrm{mbar}$ ). Organic materials are deposited using effusion cells. As cathode, deposited by thermal evaporation, aluminum on top of calcium is used. The area of the OLED pixel is $4 \mathrm{~mm}^{2}$. All measurements are performed in a glovebox system under dry $\mathrm{N}_{2}$ atmosphere at room temperature. Impedance measurements were performed using a frequency response analyzer (Solartron SI 1260 Impedance/Gain-Phase Analyzer) combined with a dielectric interface (Solartron 1296). This setup allows frequency sweeps covering a range of $10^{-3}$ to $10^{7} \mathrm{~Hz}$. The $\mathrm{AC}$ oscillator level is set to $100 \mathrm{mV} \mathrm{rms}_{\mathrm{rm}}$ for all measurements and the superimposed DC bias is varied between -4 and $+4 \mathrm{~V}$.

The usual way to characterize OLEDs electrically is to record current density - voltage $(j-V)$ and luminance - voltage $(L-V)$ characteristics, shown in fig. 2 for a device of type (2). For reverse bias and even small positive bias the leakage current is below $10^{-5} \mathrm{~mA} / \mathrm{cm}^{2}$. At a positive bias of approximately $V \approx 2 \mathrm{~V}$ for this device the current density drastically increases and the OLED starts to emit light, as can be seen in the increasing luminance value. This increase in current density is due to double injection of holes and electrons into the device at high enough positive bias forming excitons at the $\mathrm{TPD} / \mathrm{Alq}$ interface, which eventually recombine with a certain probability under emission of light. However, the quasi-static $j-V$ measurements do not reveal information about the device behavior under reverse bias conditions, i.e. below the built-in voltage. For this purpose impedance spectroscopy measurements can be used, which will be explained below.

\section{IMPEDANCE SPECTROSCOPY}

In an IS experiment, a small, alternating electrical signal $V_{\mathrm{AC}}(t)=V_{1} \cdot \sin (2 \pi f \cdot t)$ with small amplitude $V_{1}$ and frequency $f$ is applied to the sample in addition to a constant bias voltage $V_{\mathrm{DC}}=V_{0}$, resulting in a total applied voltage of

$$
V=V_{0}+V_{1} \cdot \sin (2 \pi f \cdot t) .
$$

$V_{0}$ serves as a handle to change the working point on the $j-V$ characteristics. The response of the sample is measured as the resulting current $I_{\mathrm{AC}}(t)=I_{1} \cdot \sin (2 \pi f \cdot t+\varphi)$, where $\varphi$ is a phase shift between voltage and current. The complex impedance $\hat{Z}(f)$ is defined, according to Ohm's law, as the ratio of the voltage and current response in complex notation $\hat{V}$ and $\hat{I}$, respectively, including their mutual phase shift:

$$
\hat{Z}(f)=\frac{\hat{V}}{\hat{I}}=\operatorname{Re}(\hat{Z})+\mathrm{i} \cdot \operatorname{Im}(\hat{Z}) .
$$




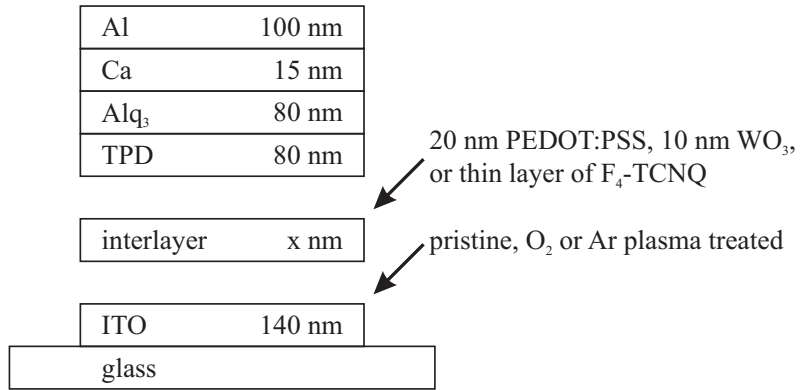

Figure 1: Bottom-emitting OLED stack with different anodes and anode treatments.

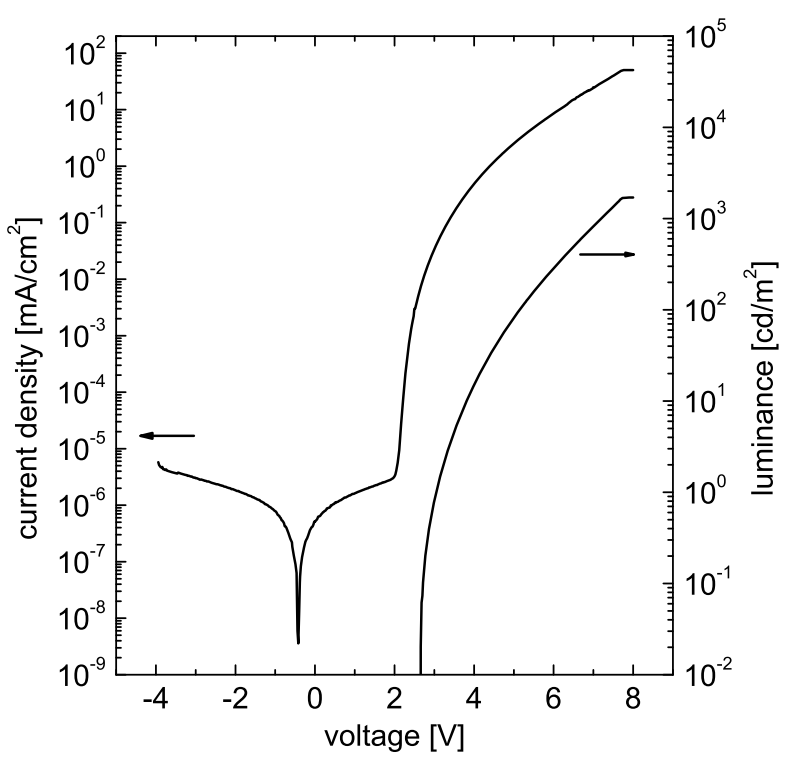

Figure 2: Current density - voltage and luminance voltage characteristic for a device of type (2). Current density limited to $50 \mathrm{~mA} / \mathrm{cm}^{2}$.

The modulus $|\hat{Z}|$ and the phase shift $\varphi$ therefore are given by

$$
|\hat{Z}|=\sqrt{\operatorname{Re}^{2}(\hat{Z})+\operatorname{Im}^{2}(\hat{Z})} \text { and } \varphi=\arctan \left(\frac{\operatorname{Im}(\hat{Z})}{\operatorname{Re}(\hat{Z})}\right),
$$

respectively. There are different equivalent representations of the complex impedance, however, the most intuitive one for semiconductor devices is the capacitance $C$, defined as

$$
C=\frac{1}{2 \pi f} \cdot \frac{-\operatorname{Im}(\hat{Z})}{\operatorname{Re}^{2}(\hat{Z})+\operatorname{Im}^{2}(\hat{Z})} .
$$

The complementary quantity is the dielectric loss (the conductance $G$ divided by the angular frequency $\omega$ )

$$
\frac{G}{\omega}=\frac{1}{2 \pi f} \cdot \frac{\operatorname{Re}(\hat{Z})}{\operatorname{Re}^{2}(\hat{Z})+\operatorname{Im}^{2}(\hat{Z})},
$$

which will not be discussed further in this paper.

The data obtained by impedance spectroscopy can be represented in several different forms, each especially suited for the topic of interest. Here we are using plots of capacitance - frequency $(C-f)$ and capacitance voltage $(C-V)$ to explain charge injection and an interfacial charge in the OLEDs, respectively. In the following two subsections the basic ideas behind these two data representations are explained.

\subsection{Capacitance of a double RC circuit}

A resistor with resistance $R$ has the (complex) impedance $\hat{Z}_{R}=R$, a capacitor with capacitance $C$ has $\hat{Z}_{C}=$ $1 /(\mathrm{i} \omega C)$ with the angular frequency $\omega=2 \pi f$. If both are combined in parallel to an RC element we obtain the impedance

$$
\hat{Z}_{R C}=\frac{1}{\frac{1}{R}+\mathrm{i} \omega C} .
$$




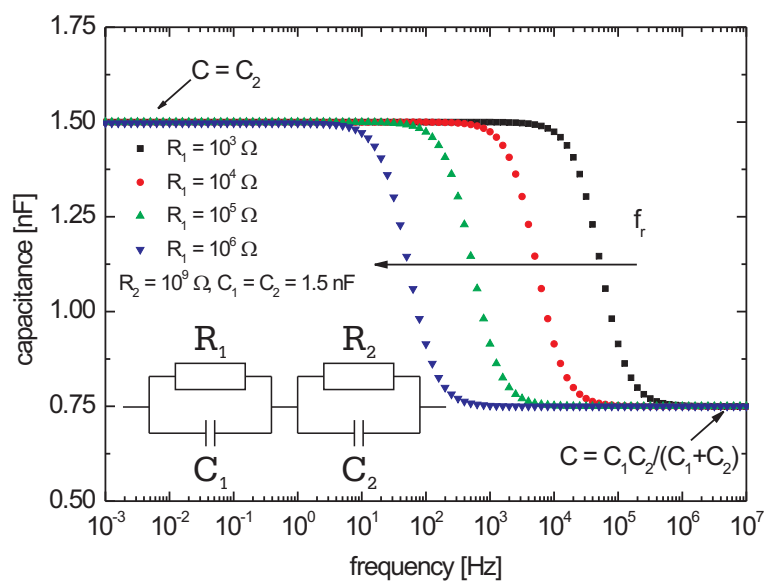

Figure 3: Capacitance - frequency plot for two RC elements in series with different values.

To model the OLED stack we consider two RC elements connected in series, representing the hole-conducting TPD layer (HTL) and the electron-conducting $\mathrm{Alq}_{3}$ layer (ETL), having the impedance

$$
\hat{Z}=\hat{Z}_{R_{1} C_{1}}+\hat{Z}_{R_{2} C_{2}}
$$

If now a voltage according to eq. 1 is applied with varying frequency $f$ we find two plateaus in the capacitance for low and high frequency and a transition region around the relaxation frequency $f_{\mathrm{r}}$ (fig. 3). According to equations 4 and 7 the limiting case for the total capacitance $C_{\text {tot }}$ in the high frequency range is

$$
\lim _{f \rightarrow \infty} C_{\text {tot }}=\frac{C_{1} C_{2}}{C_{1}+C_{2}}
$$

which is just the total capacitance of two capacitors in series. However, the low frequency limit (which corresponds to the DC regime) also depends on the values of the two resistors:

$$
\lim _{f \rightarrow 0} C_{\text {tot }}=\frac{R_{1}^{2} C_{1}+R_{2}^{2} C_{2}}{\left(R_{1}+R_{2}\right)^{2}} .
$$

The transition between these two limiting cases happens at the relaxation frequency $f_{\mathrm{r}}$ which is given by ${ }^{11}$

$$
f_{\mathrm{r}}=\frac{1}{2 \pi \tau_{\mathrm{r}}}=\frac{1}{2 \pi} \cdot \frac{R_{1}+R_{2}}{R_{1} R_{2} \cdot\left(C_{1}+C_{2}\right)} .
$$

If we now assume $R_{2} \gg R_{1}$ eq. 9 and 10 yield

$$
\lim _{f \rightarrow 0} C_{\text {tot }}=C_{2} \quad \text { and } \quad f_{\mathrm{r}}=\frac{1}{2 \pi} \cdot \frac{1}{R_{1} \cdot\left(C_{1}+C_{2}\right)}
$$

respectively. In fig. $3 R_{1}$ is varied from $10^{3}$ to $10^{6} \Omega$, while the other elements have fixed values: $R_{2}=10^{9} \Omega$ and $C_{1}=C_{2}=1.5 \cdot 10^{-9} \mathrm{~F}$. With increasing $R_{1}$ the relaxation frequency $f_{\mathrm{r}}$ shifts to lower frequencies. Observing shifts in the relaxation frequency to lower values therefore implies that a resistance in the device is increased.

\subsection{Capacitance of a TPD-Alq 3 OLED}

To model the TPD-Alq ${ }_{3}$ OLED each layer (HTL and ETL) is assigned to one RC element. As is known from literature $^{12}$ the high frequency range corresponds to the geometric capacitance $C_{\text {geo }}$ of the device

$$
C_{\mathrm{geo}}=\frac{\epsilon_{0} \epsilon_{\mathrm{r}} A}{d}
$$


where $\epsilon_{0}$ is the permittivity of free space, $\epsilon_{\mathrm{r}} \approx 3.5$ (valid for many organic materials ${ }^{12}$ ) is the relative dielectric constant, $A$ the area of the OLED pixel, and $d=d_{\mathrm{TPD}}+d_{\mathrm{Alq}_{3}}$ is the combined thickness of the HTL and ETL. The lower frequency limit is the capacitance of the $\mathrm{Alq}_{3}$ layer. The reason is that holes are injected to the TPD layer, ${ }^{12}$ making it less resistive, and accumulate at the $\mathrm{TPD} / \mathrm{Alq}_{3}$-interface, leaving just the $\mathrm{Alq}{ }_{3}$ layer as capacitor:

$$
C_{\mathrm{Alq}_{3}}=\frac{\epsilon_{0} \epsilon_{\mathrm{r}} A}{d_{\mathrm{Alq}_{3}}} .
$$

As explained in the previous subsection the relaxation frequency $f_{\mathrm{r}}$ yields information about the resistivity of the HTL: if $f_{\mathrm{r}}$ is shifted to smaller frequencies the resistivity becomes higher. Thus capacitance - frequency $(C-f)$ sweeps allow the study of charge injection properties for OLEDs. An example for $C-f$ measurements of a device of type (2) is shown in fig. 4 for different applied DC bias. The rise in capacitance at very low and very high frequencies will be addressed in the next section. In reverse bias condition $\left(V_{0}=-2 \mathrm{~V}\right)$ no holes are injected, the capacitance is the total geometric capacitance $(C \approx 0.75 \mathrm{nF})$ of the device. Increasing the bias voltage $\left(V_{0}=0 \mathrm{~V}\right.$ and $\left.2 \mathrm{~V}\right)$ yields the two plateaus discussed above with the relaxation frequency $f_{\mathrm{r}}$ in the order of $10^{4}-10^{5} \mathrm{~Hz}$. In forward bias condition $\left(V_{0}=4 \mathrm{~V}\right)$, when the OLED emits light, the step at the relaxation frequency is still observeable, as it corresponds to the injection of holes into the HTL. However, for low enough frequencies also electrons are injected into the device. As they recombine with the holes, charge is annihilated and as consequence the capacitance of the device decreases.

The resistivity of the HTL is changing with applied bias as it becomes "conducting". Therefore, in combination with the $C-f$ measurements, another impedance spectroscopy measurement is very useful for studying the electronic properties of OLEDs: capacitance - voltage measurements $(C-V)$. Here, the bias voltage $V_{0}$ is varied for assorted frequencies $f$. An example is given in fig. 5, again for the type (2) device. At very large negative bias we see a constant capacitance, corresponding to the geometric capacitance (eq. 12) of the device. However, if the bias becomes larger than a transition voltage $V_{\mathrm{t}}$ holes are injected to the device, raising the capacitance, as they accumulate at the TPD $/ \mathrm{Alq}_{3}$-interface. At the built-in voltage $V_{\mathrm{bi}}$, electrons are also injected to the device and the OLED starts to emit light*. As electrons and holes recombine charge is annihilated, thus the capacitance decreases rapidly with increasing potential. The overall behavior requires that a negative interfacial charge $Q_{\text {if }}$ is present at the TPD/Alq ${ }_{3}$-interface ${ }^{10,12}$ (as shown in the energy diagram in fig. 6). At $V_{0}<V_{\mathrm{t}}$ (point (a) in fig. 5) both TPD and $\mathrm{Alq}_{3}$ are reverse biased and no charges can be injected. Increasing the voltage reduces the slope, until the flat-band case for TPD is reached $\left(V_{0}=V_{\mathrm{t}}\right.$, point $\left.(\mathrm{b})\right)$, holes are injected. However, as an effect of the negative interfacial charge the bands in $\mathrm{Alq}_{3}$ are not yet in the flat-band case, meaning that no electrons can be injected at this point. More and more holes can be injected with increasing potential, raising the capacitance of the device $\left(V_{\mathrm{t}}<V_{0}<V_{\mathrm{bi}}\right.$, point (c)), while the slope of the bands in $\mathrm{Alq}_{3}$ is decreasing. Finally, at $V_{0}=V_{\mathrm{bi}}$ (point (d)) the flat-band condition for $\mathrm{Alq}_{3}$ is reached and electrons can be injected to the device. For even higher voltages $V_{0}>V_{\mathrm{bi}}$ (point (e)) the capacitance of the OLED decreases rapidly, as holes and electrons recombine, annihilating charge.

If there would be no interfacial charge the threshold voltage and the built-in voltage would be equal $\left(V_{\mathrm{t}}=V_{\mathrm{bi}}\right)$, as the flat-band case would be reached simultaneously in the TPD and the $\mathrm{Alq}_{3}$ layer (simultaneous hole and electron injection). As we clearly see that $V_{\mathrm{t}} \neq V_{\mathrm{bi}}$ we come to the conclusion that a negative interfacial charge density $\sigma_{\text {if }}$ is present. Its value can be calculated from the following equation: ${ }^{12}$

$$
\sigma_{\mathrm{if}}=Q_{\mathrm{if}} / A=\frac{\epsilon_{0} \epsilon_{\mathrm{r}}}{d_{\mathrm{Alq}_{3}}} \cdot\left(V_{\mathrm{t}}-V_{\mathrm{bi}}\right) .
$$

With $\epsilon_{\mathrm{r}} \approx 3.5, A=4 \mathrm{~mm}^{2}$ and $d_{\mathrm{Alq}_{3}}=80 \mathrm{~nm}$ we obtain $Q_{\mathrm{if}} \approx-5.1 \mathrm{nC}$ and $\sigma_{\mathrm{if}} \approx-1.3 \mathrm{mC} / \mathrm{m}^{2}$. Similar values, also for other material combinations and obtained with other measurement techniques, have been published previously. ${ }^{10,13-15}$ Recent studies show that the origin of this negative charge at the interface is most likely due to dipole moment ordering in the $\mathrm{Alq}_{3}$ layer during film preparation. ${ }^{15}$

${ }^{*}$ Strictly speaking, one has to distinguish between the detected onset voltage $V_{\text {on }}$ of the current or the luminance, the peak voltage $V_{\text {peak }}$ observed in $C-V$ measurements and the built-in voltage $V_{\mathrm{bi}}$ given by the difference in work function of the anode and cathode material. As pointed out in a recent publication by van Mensfoort et al. ${ }^{8}$ due to the contribution of diffusion currents and disorder the experimental quantities $V_{\text {on }}$ and $V_{\text {peak }}$ can be lower than $V_{\text {bi }}$ by some tenths of a volt. However, for the sake of simplicity we will not make this distinction here.

7415-15 V. 3 (p.5 of 12) / Color: No / Format: A4 / Date: 7/10/2009 10:48:43 AM 


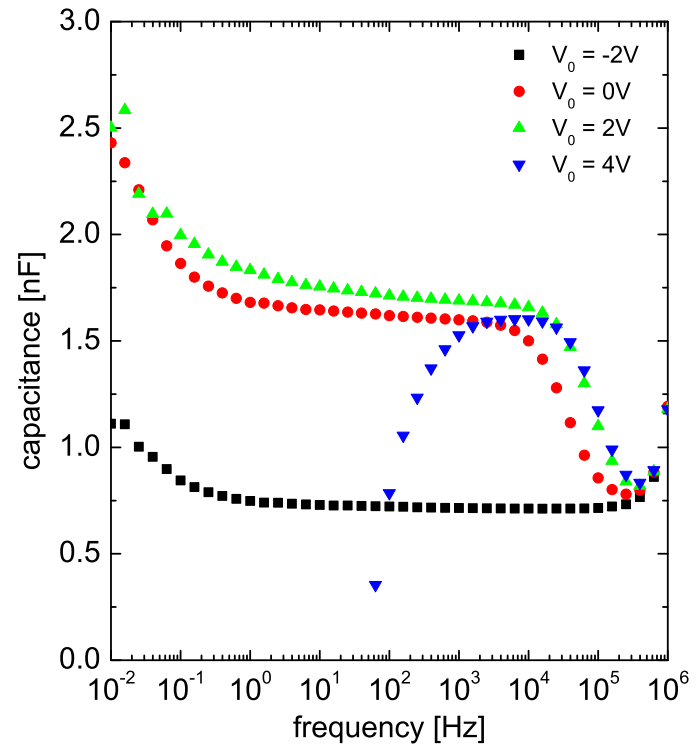

Figure 4: Capacitance - frequency plot for the OLED of type (2) at different applied bias $V_{0}=-2 \mathrm{~V}, 0 \mathrm{~V}$, $2 \mathrm{~V}$, and $4 \mathrm{~V}$.

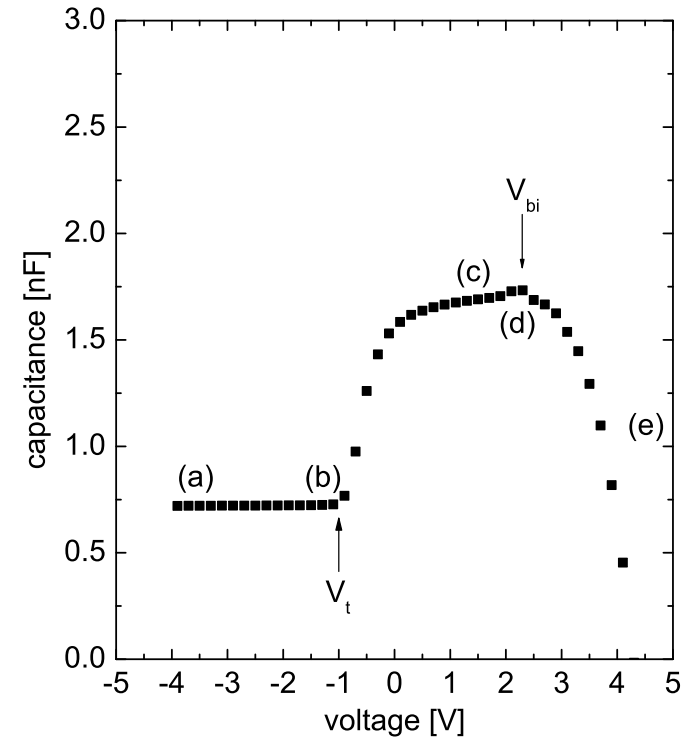

Figure 5: Capacitance - voltage plot for the OLED of type (2) at fixed frequency $f=100 \mathrm{~Hz}$. Letters (a) to (e) correspond to the band diagrams discussed in fig. 6 .

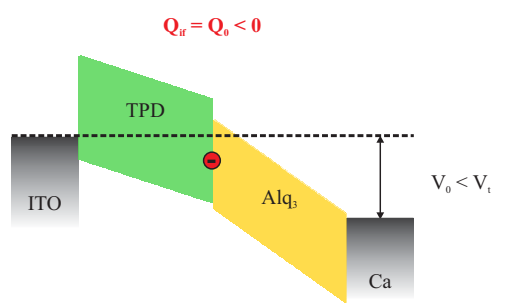

(a) $V_{0}<V_{\mathrm{t}}$ : no charge injection.

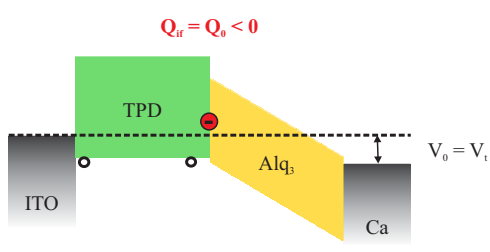

(b) $V_{0}=V_{\mathrm{t}}$ : holes start to inject from the ITO electrode (flat band condition in the TPD layer).

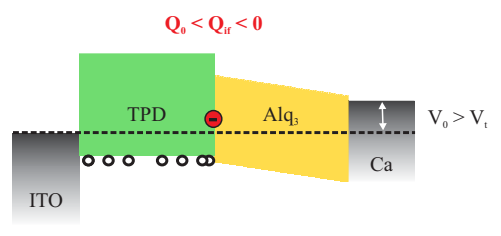

(c) $V_{\mathrm{t}}<V_{0}<V_{\mathrm{bi}}:$ more holes are injected and accumulate at the $\mathrm{TPD} / \mathrm{Alq}_{3}$-interface.

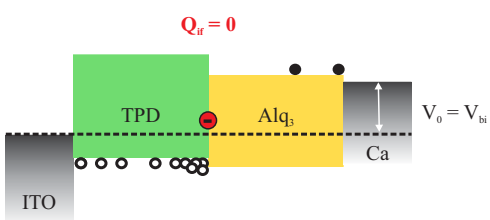

(d) $V_{0}=V_{\mathrm{bi}}$ : electrons start to inject from the $\mathrm{Ca}$ electrode (flat band condition in the $\mathrm{Alq}_{3}$ layer).

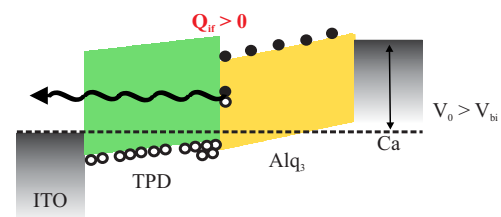

(e) $V_{0}>V_{\mathrm{bi}}$ : more electrons are injected into the OLED and recombine with the holes under emission of light.

Figure 6: Simplified band diagrams for different applied bias voltage $V_{0}$. 


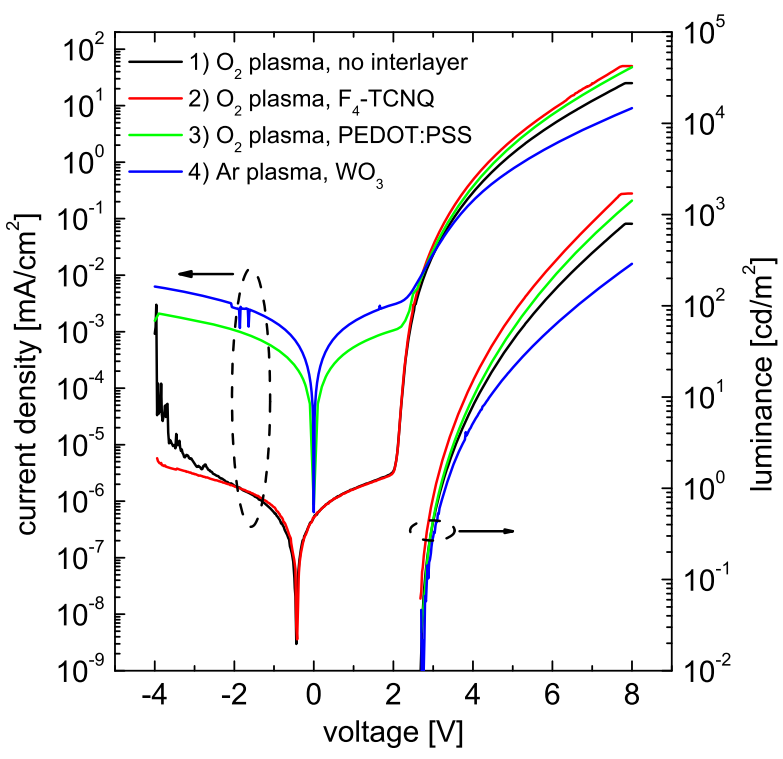

Figure 7: Current density - and luminance - voltage plots for the four anode modifications of the OLED presented in fig. 1. Higher leakage current $(-4 \mathrm{~V}<$ $V<2 \mathrm{~V})$ for the devices with PEDOT:PSS and $\mathrm{WO}_{3}$ possibly due to lateral conduction (additional current paths). Current density limited to $50 \mathrm{~mA} / \mathrm{cm}^{2}$.

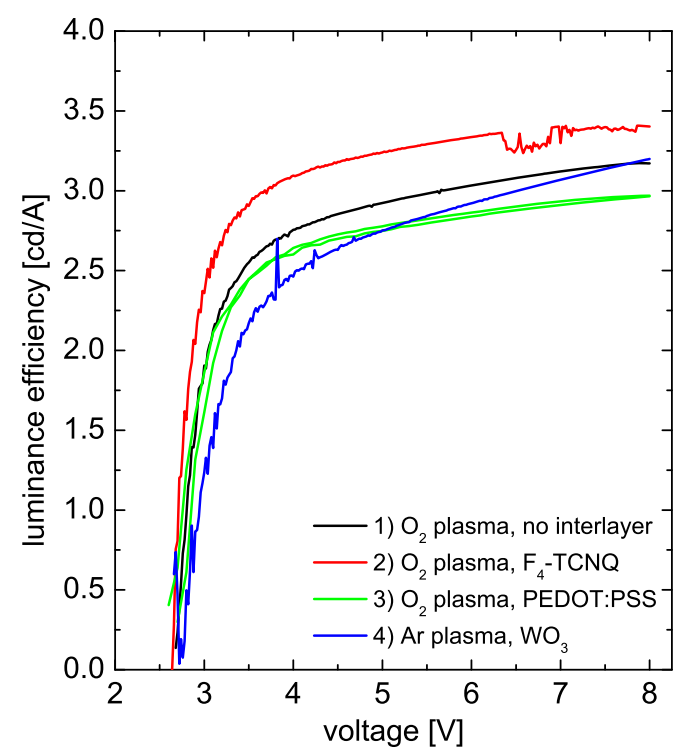

Figure 8: Luminance efficiency for the four anode modifications of the OLED presented in fig. 1.

\section{INFLUENCE OF DIFFERENT ANODES}

In this paper we compare four different anodes (table 1) and their influence on the electronic properties of the OLED stack presented in fig. 1. The $j-V$ and $L-V$ characteristics are shown in fig. 7, as well as the luminance efficiency (i.e. luminance / current density) in fig. 8. All current densities below $V_{\mathrm{bi}}=2 \mathrm{~V}$ are considered as leakage currents, since no light is emitted from the OLED. Devices (1) and (2) show similar leakage current densities, basically $j<10^{-5} \mathrm{~mA} / \mathrm{cm}^{2}$. The leakage currents for devices (3) and (4) are about three orders of magnitude higher, device (4) being worse than device (3). This might be due to additional current paths due to lateral conduction of the interlayers. Under forward bias $\left(V>V_{\mathrm{bi}}\right)$ devices (1-3) show almost the same current densities. Device (4), with $\mathrm{WO}_{3}$, on the other side shows slightly smaller current densities, which results in lower luminance values than for the other devices.

We now analyze these OLEDs with $C-f$ measurements (fig. 9). At a bias of $V_{0}=0 \mathrm{~V}$ devices (1-3) show a relaxation frequency at approximately $4 \cdot 10^{4} \mathrm{~Hz}$ and device (4) at $6 \cdot 10^{3} \mathrm{~Hz}$. This means that hole injection in device (4) is not as good as in the other three devices, which explains the lower current densities observed in the $j-V$ measurement. From this IS result one can come to the conclusion that device (4), with Ar treated ITO and $\mathrm{WO}_{3}$ anode, is not the best choice for this OLED stack, even though the current density is not so much different compared to the other devices. Together with the $j-V$ data (fig. 7) device (2), with $\mathrm{O}_{2}$ treated ITO and $\mathrm{F}_{4}$-TCNQ interlayer, would be the best choice of the four anodes studied here: high relaxation frequency $f_{\mathrm{r}}$ (good hole injection), low leakage current, and highest luminance efficiency.

However, there are several different features visible in the $C-f$ data which can not be described by the theory of two RC elements in series that would lead to two constant plateaus already shown in fig. 3 for the forward bias condition. The rise in capacitance above $f \approx 2 \cdot 10^{5} \mathrm{~Hz}$ is due to very long cables (ca. $12 \mathrm{~m}$ ) used to connect our sample inside the glovebox system. Using short cables avoids this problem. This rise in capacitance at high frequencies therefore is not a feature of the devices under test and is not considered here. The second step in capacitance at low frequency $\left(f<f_{\mathrm{r}}\right)$ can be modelled with three RC elements in series, however, there 


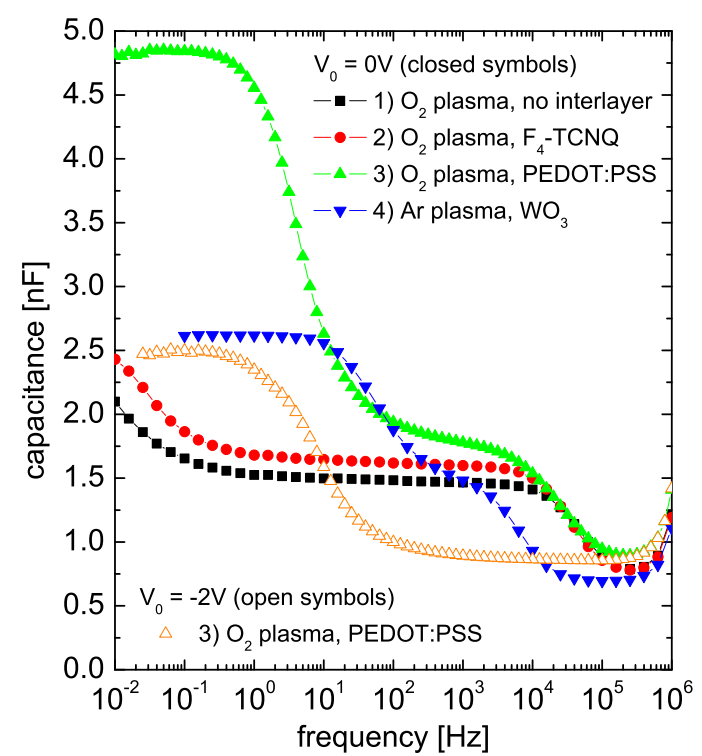

Figure 9: Capacitance - frequency plot for the four anode modifications of the OLED presented in fig. 1. Bias voltage closed symbols: $V_{0}=0 \mathrm{~V}$, open symbols: $V_{0}=-2 \mathrm{~V}$. Shift in relaxation frequency $f_{\mathrm{r}}$ for device (4): worse hole injection. Rise in capacitance at high frequencies: effect of long cables used to connect the OLEDs. Rise in capacitance for low frequency: possibly due to lateral conduction (see text).

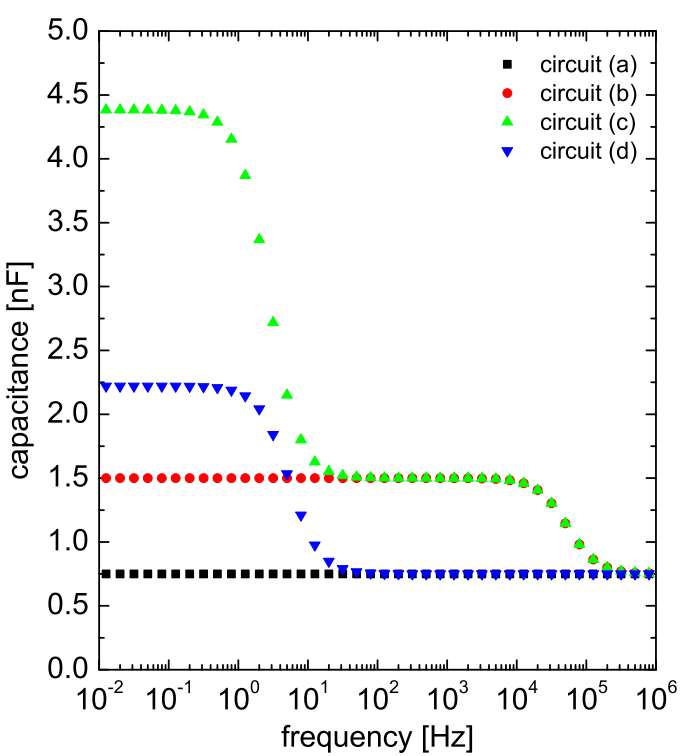

Figure 10: Simulated capacitances for the models shown in fig. 11. Black squares: ideal OLED in reverse bias. Red circles: ideal OLED in forward bias. Green triangles pointing upwards: OLED with additional current path in forward bias. Blue triangles pointing downwards: OLED with additional current path in reverse bias.

is no physical counterpart for a third RC element in the device. A more probable approach is indicated by the leakage currents: due to the lateral conduction of the layers on the anode side there exists an additional current path through the device. The surrounding of the actual OLED pixel has to be taken into account. In fig. 10 we simulated the OLED behavior for four model circuits shown in fig. 11. Circuit (a) (black squares) corresponds to the OLED under reverse bias $\left(V<V_{\mathrm{r}}\right)$ where no charge carriers are injected, thus both resistances are very high. The frequency behavior shows only the constant capacitance of the two capacitors in series. Circuit (b) (red circles) corresponds to an OLED into which holes can be injected, thus $R_{1} \ll R_{2}$. Here we observe the two constant plateaus already shown earlier. We now allow an additional current path through the device, still in forward bias (circuit (c)): two additional RC elements in series with a resistor, alltogether in parallel to the original $\mathrm{RC}$ elements. The values for the additional $\mathrm{RC}$ elements are in the same order as the original ones, as they also correspond to the TPD and $\mathrm{Alq}_{3}$ layers, respectively. The curve (green triangles facing upwards) now shows an additional step at low frequency, which is similar to the one measured for the device with PEDOT:PSS (green triangles in fig. 9). The higher the leakage current the higher this additional step at low frequency. Another indication that the additional current path plays an important role for the low frequency behavior of OLEDs is given by circuit (d) (blue triangles facing downwards), where the OLED is simulated with the additional path under reverse bias: a step at about $5 \mathrm{~Hz}$ occurs, which is at a similar frequency as the second step under forward bias (circuit (c)). This step is also seen in the $C-f$ data of the PEDOT:PSS device (3) under reverse bias (open orange triangles in fig. 9). This confirms the validity of the models with additional current path due to lateral conduction of the interlayers.

\section{DEVICE DEGRADATION}

It is generally known that the luminance of OLEDs is decreasing with operating time and current load, hence their lifetime unfortunately is still limited. There have been various reports about possible degradation mechanisms, 


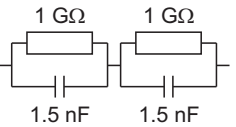

(a) Two RC elements with high resistances: no charge injection (reverse bias condition).

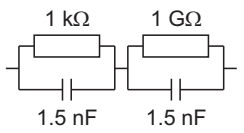

(b) Two RC elements with different resistances (holes are injected).

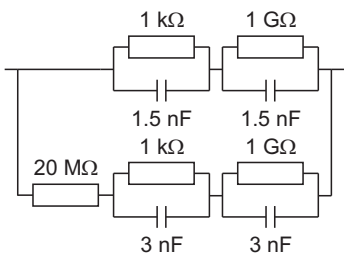

(c) Additional current path due to lateral conduction, forward bias condition (holes are injected).

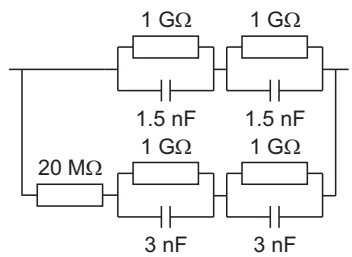

(d) Additional current path due to lateral conduction, reverse bias condition.

Figure 11: Models used for the simulations of the capacitance - frequency behavior shown in fig. 10.

e.g. migration of mobile ions in the device, ${ }^{16}$ reorientation of molecular dipoles,${ }^{17,18}$ chemical degradation due to oxygen ${ }^{19}$ and/or water ${ }^{20}$ or instability of charge-carrying species (e.g. $\left.\mathrm{Alq}_{3}\right) .{ }^{21}$ However, as these reports use different materials and material combinations it might be that one or the other mechanisms is more pronounced in the OLED stack of interest.

Again, impedance spectroscopy is a handy tool to contribute to these studies. Here we are going to use IS on an OLED stack of type (4), with Ar treated ITO and $10 \mathrm{~nm} \mathrm{WO}_{3}$ interlayer. To degrade the device a constant current density of $j=10 \mathrm{~mA} / \mathrm{cm}^{2}$, which is quite low, has been applied, while the voltage $V$ and luminance $L$ have been recorded, see fig. 12. Aging has been performed in a nitrogen glovebox in dark and at room temperature, which was slightly changing over time, however, effects due to different temperature of the sample can be excluded due to only small variations $\left( \pm 1^{\circ} \mathrm{C}\right)$. Aging has been stopped after 10 hours and $j-V, C-f$, and $C-V$ measurements have been performed. The measurements have not been carried out subsequently in all cases, sometimes the OLED was stored for a couple of days unused in the glovebox before intentional aging was continued.

During degradation there are reversible and irreversible effects on the OLED's electrical behavior: the driving voltage increases, however, the device seems to recover after not being operated for some time, as the voltage restarts at smaller values when the device is artificially degraded again. This effect also affects the luminance, which increases again after "regeneration", however this is less pronounced than the recovery in voltage. Such effects have already been reported for expample by Yahiro et al. ${ }^{18}$ Fig. 13 shows the current density - voltage plots for the different aging times. It shows that the built-in voltage $V_{\mathrm{bi}}$ is unchanged. The effect of electrical aging of the device on the current density is only very small: at $6 \mathrm{~V}$ only a change of about $24 \%$. Just from this $j-V$ curve one would expect that the device has not aged significantly. However, the variation in luminance is much more pronounced: a change of about $46 \%$ is observed, again at $6 \mathrm{~V}$.

We also applied IS measurements on this electrically aged device. Fig. 14 shows the capacitance - frequency behavior. A pronounced shift in the relaxation frequency to lower frequencies is observed with aging time. Therefore the HTL resistance is increasing during aging. In the capacitance - voltage plots (fig. 15) one observes that the built-in potential $V_{\mathrm{bi}}$ is not shifted, as is already expected from the $j-V$ measurement (fig. 13). However, the transition voltage $V_{\mathrm{t}}$ shifts to more positive potential with aging time. This means that the absolute value of the (negative) interfacial charge density gets smaller. The reduction of the interfacial charge density might be due to the generation of positive charge near the HTL/ETL-interface. This charge could be due to hole traps, which act as nonradiative recombination centers, ${ }^{14}$ thus the luminance of the device is decreasing. As the positive charge is increasing with aging time, more nonradiative recombination centers are built, reducing the luminance significantly. Plotting the luminance efficiency versus the transition voltage (or the charge density) results in a linear relationship (fig. 16), correlating the generation of (positive) charges with loss in luminance.

However, not only the model of immobile charges $^{14,22}$ offers an explanation for the observed degradation effects. Indium migration from the ITO electrode into the emitting $\mathrm{Alq}_{3}$ layer could also play a role, ${ }^{16,23}$ but it has been shown rather convincingly by Luo and Popovic et al. that its effect is not dominant. ${ }^{24}$ They give another explanation for generated positive charges: the generation of $\mathrm{Alq}_{3}$ cation-radicals upon degradation ${ }^{21,24,25}$ due to hole current through the $\mathrm{Alq}_{3}$ layer. This cationic $\mathrm{Alq}_{3}$ species are supposed to be leading to the formation 


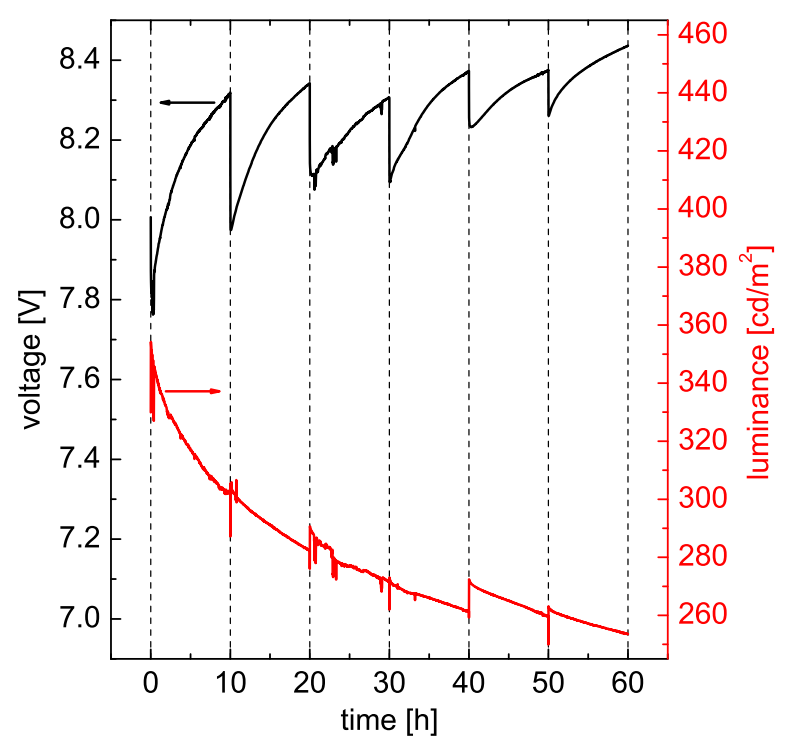

Figure 12: Artificial degradation of an OLED stack with $\mathrm{Ar}$ treated ITO and $10 \mathrm{~nm} \mathrm{WO}_{3}$ interlayer (fig. 1). Current density $j=10 \mathrm{~mA} / \mathrm{cm}^{2}$.

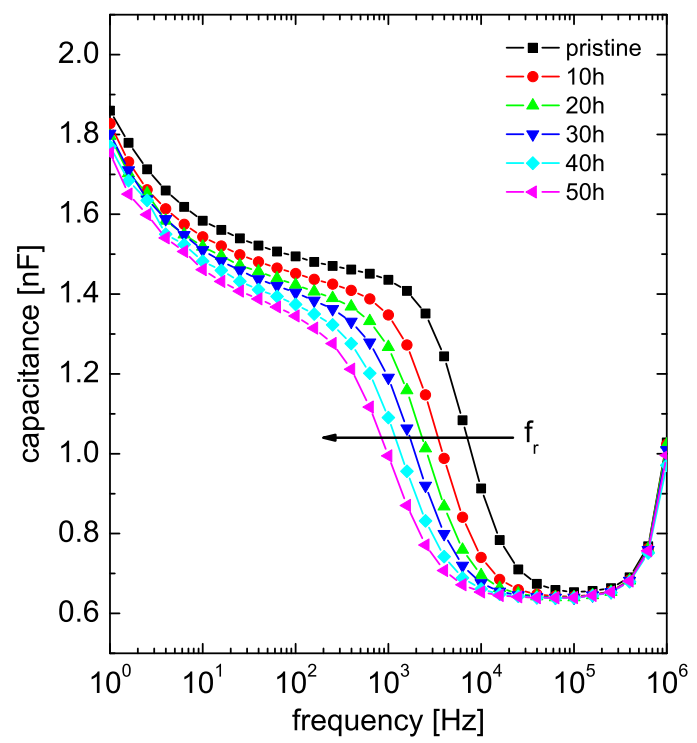

Figure 14: Capacitance - frequency plots for the different aging times. Applied bias: $V_{0}=2 \mathrm{~V}$. Relaxation frequency $f_{\mathrm{r}}$ shifts to lower frequencies with aging time.

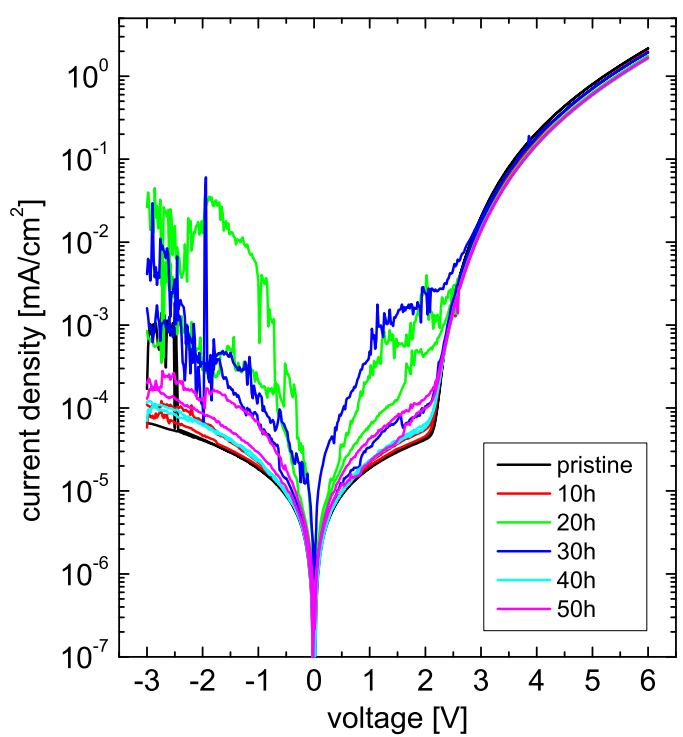

Figure 13: Current density - voltage plot for the different aging times. $V_{\mathrm{bi}}$ unchanged. Only small variation in current density in forward bias $(24 \%$ at $6 \mathrm{~V})$.

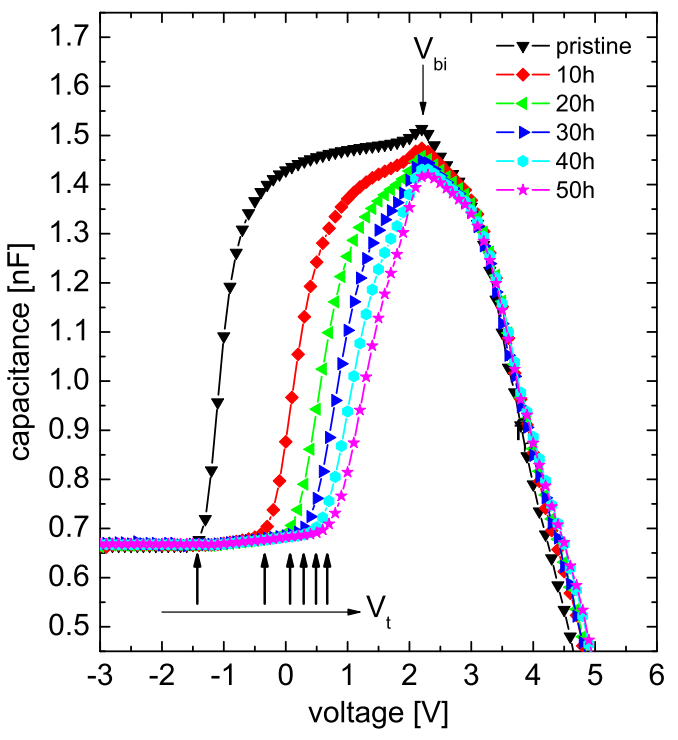

Figure 15: Capacitance - voltage plots for the different aging times. Applied frequency: $f=100 \mathrm{~Hz} . V_{\mathrm{bi}}$ is not changing, $V_{\mathrm{t}}$ shifts to more positive voltages with aging time. 
of fluorescence quenchers. Alternatively, degradation of the HTL close to the HTL/ETL interface was suggested as the origin of device degradation. ${ }^{26}$ Thus, further systematic studies are needed and IS offers the possibility to contribute to the ongoing discussions.

\section{SUMMARY}

In this paper we have used impedance spectroscopy to study charge injection in OLEDs with different anodes and degradation processes. We have shown that capacitance - frequency analysis is a sensitive tool to monitor charge injection and yields information especially in voltage regimes $\left(V<V_{\mathrm{bi}}\right)$, where current density - voltage measurements are not sensitive. Capacitance - voltage analysis shows trapped or interfacial charges. In the OLED stack investigated here, a negative interfacial charge is present at the $\mathrm{TPD} / \mathrm{Alq}_{3}$-interface. However, its amount is changing with the operational lifetime of the OLED, which is concluded from $C-V$ measurements. Therefore, both types of measurements presented in this paper are very useful tools in the analysis of degradation processes in OLEDs.

\section{ACKNOWLEDGMENTS}

The authors would like to thank the German Federal Ministry of Education and Research (BMBF) for funding part of this work under contract FKZ 13N10474 (TOPAS). We also thank Y. Noguchi (Chiba University, Japan) for fruitful discussions.

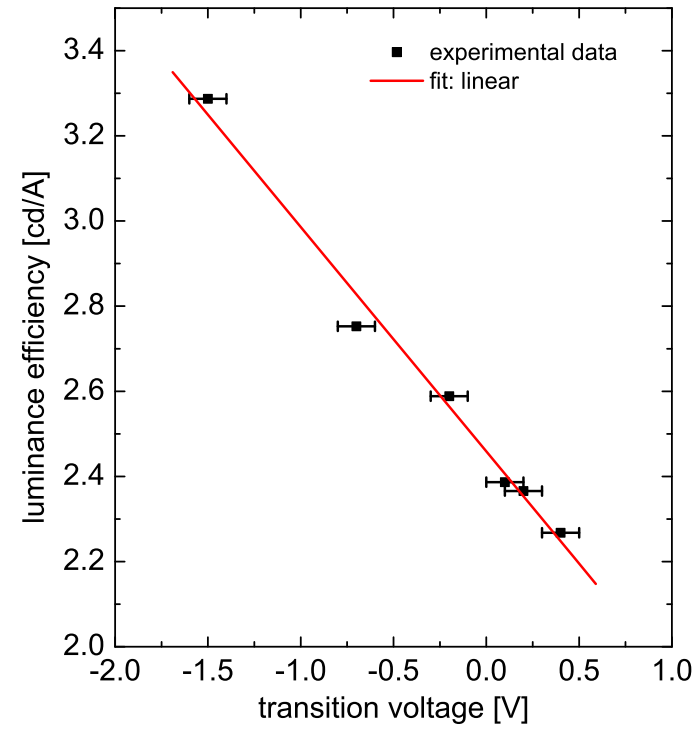

Figure 16: Luminance efficiency loss as a function of transition voltage. Black squares: experimental data. Red line: linear fit.

\section{REFERENCES}

[1] Macdonald, J. R., ed., [Impedance Spectroscopy], John Wiley \& Sons (1987).

[2] Garrido, J. A., Nowy, S., Härtl, A., and Stutzmann, M., "The diamond/aqueous electrolyte interface: an impedance investigation," Langmuir 24(8), 3897 - 3904 (2008).

[3] Meier, M., Karg, S., and Riess, W., "Light-emitting diodes based on poly-p-phenylene-vinylene: II. Impedance spectroscopy," Journal of Applied Physics 82(4), 1961 - 1966 (1997).

[4] Scherbel, J., Nguyen, P. H., Paasch, G., Brütting, W., and Schwoerer, M., "Temperature dependent broadband impedance spectroscopy on poly-(p-phenylene-vinylene) light-emitting diodes," Journal of Applied Physics 83(10), 5045 - 5055 (1998).

[5] Campbell, A. J., Bradley, D. D. C., Werner, E., and Brütting, W., "Deep level transient spectroscopy (DLTS) of a poly(p-phenylene vinylene) schottky diode," Synthetic Metals 111-112, 273 - 276 (2000).

[6] Martens, H. C. F., Brom, H. B., and Blom, P. W. M., "Frequency-dependent electrical response of holes in poly(p-phenylene vinylene)," Physical Review B 60(12), R8489 - R8492 (1999).

[7] Berleb, S. and Brütting, W., "Dispersive electron transport in tris(8-hydroxyquinoline) aluminum ( $\left.\mathrm{Alq}_{3}\right)$ probed by impedance spectroscopy," Physical Review Letters 89(28), 286601 (2002).

[8] van Mensfoort, S. L. M. and Coehoorn, R., "Determination of injection barriers in organic semiconductor devices from capacitance measurements," Physical Review Letters 100, 086802 (2008).

[9] Ehrenfreund, E., Lungenschmied, C., Dennler, G., Neugebauer, H., and Sariciftci, N. S., "Negative capacitance in organic semiconductor devices: Bipolar injection and charge recombination mechanism," Applied Physics Letters 91, 012112 (2007).

[10] Berleb, S., Brütting, W., and Paasch, G., "Interfacial charges and electric field distribution in organic hetero-layer light-emitting devices," Organic Electronics 1, 41 - 47 (2000). 
[11] Taylor, D. M. and Gomes, H. L., "Electrical characterization of the rectifying contact between aluminium and electrodeposited poly(3-methylthiophene)," Journal of Physics D - Applied Physics 28, 2554- 2568 (1995).

[12] Brütting, W., Berleb, S., and Mückl, A. G., "Device physics of organic light-emitting diodes based on molecular materials," Organic Electronics 2, 1 - 36 (2001).

[13] Brütting, W., Riel, H., Beierlein, T., and Riess, W., "Influence of trapped and interfacial charges in organic multilayer light-emitting devices," Journal of Applied Physics 89, 1704 - 1712 (2001).

[14] Kondakov, D. Y., Sandifer, J. R., Tang, C. W., and Young, R. H., "Nonradiative recombination centers and electrical aging of organic light-emitting diodes: Direct connection between accumulation of trapped charge and luminance loss," Journal of Applied Physics 93(2), 1108 - 1119 (2003).

[15] Noguchi, Y., Sato, N., Tanaka, Y., Nakayama, Y., and Ishii, H., "Threshold voltage shift and formation of charge traps induced by light irradiation during the fabrication of organic light-emitting diodes," Applied Physics Letters 92, 203306 (2008).

[16] Lee, S. T., Gao, Z. Q., and Hung, L. S., "Metal diffusion from electrodes in organic light-emitting diodes," Applied Physics Letters 75(10), $1404-1406$ (1999).

[17] Yamada, T., Zou, D., Jeong, H., Akaki, Y., and Tsutsui, T., "Recoverable degradation and internal field forming process accompanied by the orientation of dipoles in organic light emitting diodes," Synthetic Metals 111-112, 237 - 240 (2000).

[18] Yahiro, M., Zou, D., and Tsutsui, T., "Recoverable degradation phenomena of quantum efficiency in organic EL devices," Synthetic Metals 111-112, 245 - 247 (2000).

[19] Scott, J. C., Kaufman, J. H., Brock, P. J., DiPetro, R., Salem, J., and Goitia, J. A., "Degradation and failure of MEH-PPV light-emitting diodes," Journal of Applied Physics 79(5), 2745 - 2751 (1996).

[20] Papadimitrakopoulos, F., Zhang, X.-M., Thomsen, D. L., and Higginson, K. A., "A chemical failure mechanism for aluminum(III) 8-hydroxyquinoline light-emitting devices," Chemistry of Materials 8, 1363 - 1365 (1996).

[21] Aziz, H., Popovic, Z. D., Hu, N.-X., Hor, A.-M., and Xu, G., "Degradation mechanism of small moleculebased organic light-emitting devices," Science 283, 1900 - 1902 (1999).

[22] Kondakov, D. Y., "Direct observation of deep electron traps in aged organic light emitting diodes," Journal of Applied Physics 97, 024503 (2005).

[23] Vestweber, H. and Rieß, W., "Highly efficient and stable organic light-emitting diodes," Synthetic Metals 91, $181-185$ (1997).

[24] Luo, Y., Aziz, H., Popovic, Z. D., and Xu, G., "Degradation mechanisms in organic light-emitting devices: Metal migration model versus unstable tris(8-hydroxyquinoline) aluminum cationic model," Journal of Applied Physics 101, 034510 (2007).

[25] Popovic, Z. D., Aziz, H., Hu, N.-X., Ioannidis, A., , and dos Anjos, P. N. M., "Simultaneous electroluminescence and photoluminescence aging studies of tris(8-hydroxyquinoline) aluminum-based organic lightemitting devices," Journal of Applied Physics 89(8), 4673 - 4675 (2001).

[26] Kondakov, D. Y., "Role of chemical reactions of arylamine hole transport materials in operational degradation of organic light-emitting diodes," Journal of Applied Physics 104, 084520 (2008). 